\section{Endoscopic ultrasound-guided duodenojejunal anastomosis to treat postsurgical Roux-en-Y hepaticojejunostomy stricture: a dream or a reality?}

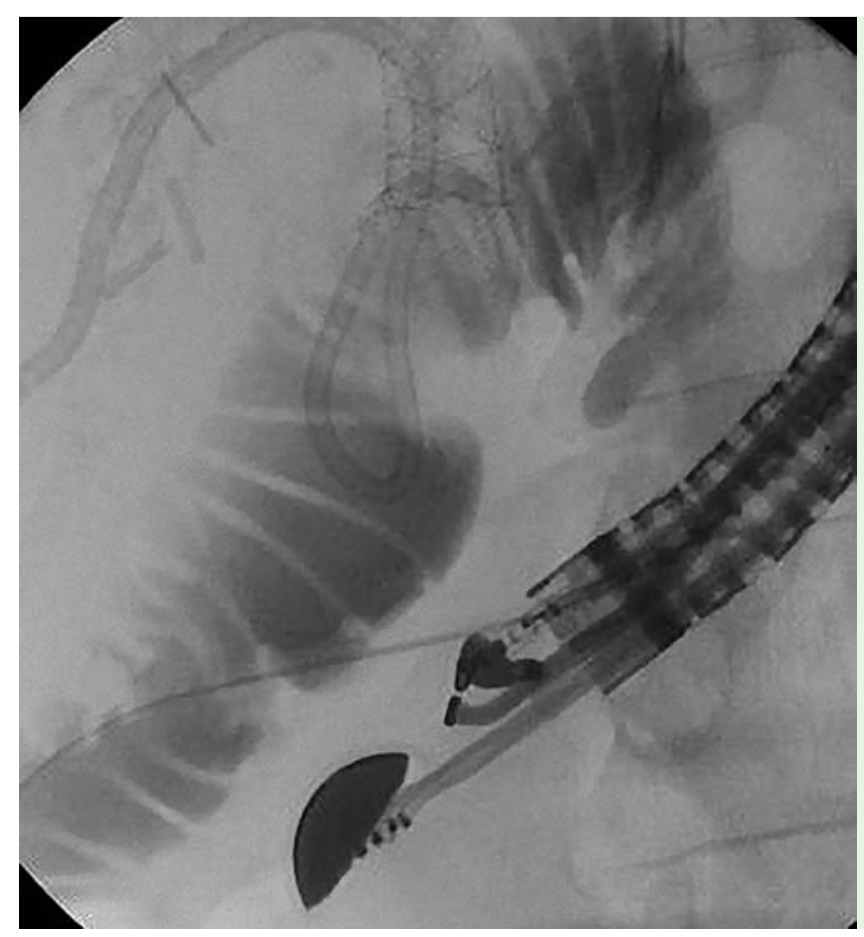

Fig. 1 Radiologic view of endoscopic ultrasound-guided puncture of the afferent loop in a 67-yearold man with recurrent cholangitis due to benign stricture of a bilioenteric anastomosis. The patient had a history of sclerosing cholangitis and left hepatectomy with Roux-en-Y bilioenteric reconstruction.

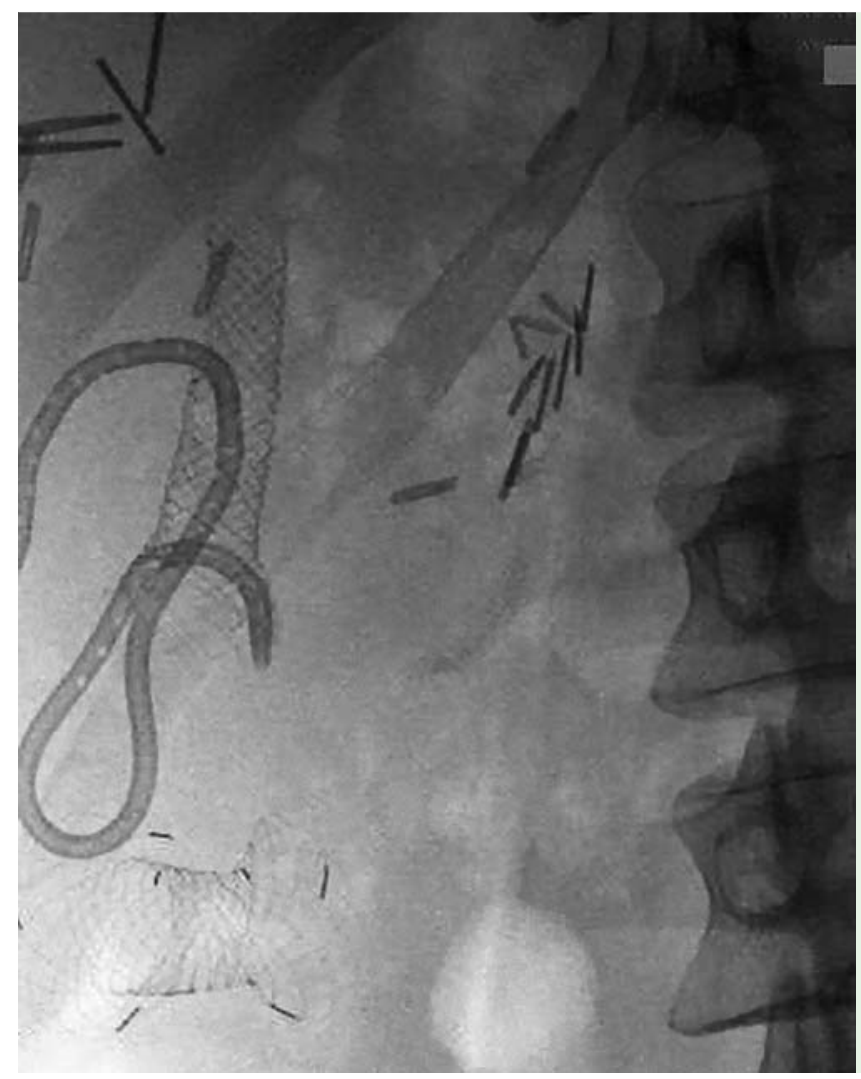

Roux-en-Y hepaticojejunostomy stricture develops in $8 \%$ to $40 \%$ of patients, and reintervention is frequently required $[1,2]$. Surgery remains the gold standard to treat this problem. We present the case of a 67-year-old man with recurrent cholangitis due to benign stricture of a bilioenteric anastomosis. The patient had a history of sclerosing cholangitis and left hepatectomy with Roux-en-Y bilioenteric reconstruction.

In another hospital, the patient had been treated via a percutaneous trans-hepatic approach with an uncovered self-expandable metal stent (u-SEMS). Biliary lithiasis and several episodes of cholangitis recurred soon thereafter. Many attempts were made to remove the stones radiologically, but they all failed. Percutaneous internalexternal drainage was then placed temporarily, and the patient was referred to our tertiary endoscopic center.

A peroral endoscopic procedure with a rendezvous technique was attempted by passing a guidewire through the internal-external drainage, but it did not succeed because the anastomotic loop was too long and tortuous. Therefore, a new endoscopic approach with an endoscopic ultrasound (EUS)-guided transenteric anastomosis was used. After the injection of contrast medium into the jejunal loop, an EUS-guided puncture was performed from the duodenal portion with a 19-gauge needle (ECHO-19, Cook Medical) ( Fig.1); a 0.035-guidewire was then placed into the jejunal loop, and a duodenojejunal fistula was created by pushing an 8.5-Fr cystoenterostome (XS 1341, Endoflex) on the guidewire. Finally, a 20-mm-long and 16-mm-diameter uSEMS (Nagi stent; Taewoong Medical) was left in place to allow consolidation of the endoscopic fistula ( $\mathbf{F i g} .2$ ). After 3 days, an operative gastroscope (Pentax) was advanced through the stent to perform endoscopic retrograde cholangiopancreatography (ERC) and remove the previous uSEMS ( $\bullet$ Fig.3). The patient was discharged after 2 days. No complications or procedure-related symptoms have been reported during 12 months of clinical follow-up.

EUS-guided duodenojejunal anastomosis is a feasible endoscopic approach in selected patients $[3,4]$. Expert and skilled endoscopists are needed to perform the described procedure successfully.

Endoscopy_UCTN_Code_TTT_1AS_2AB 


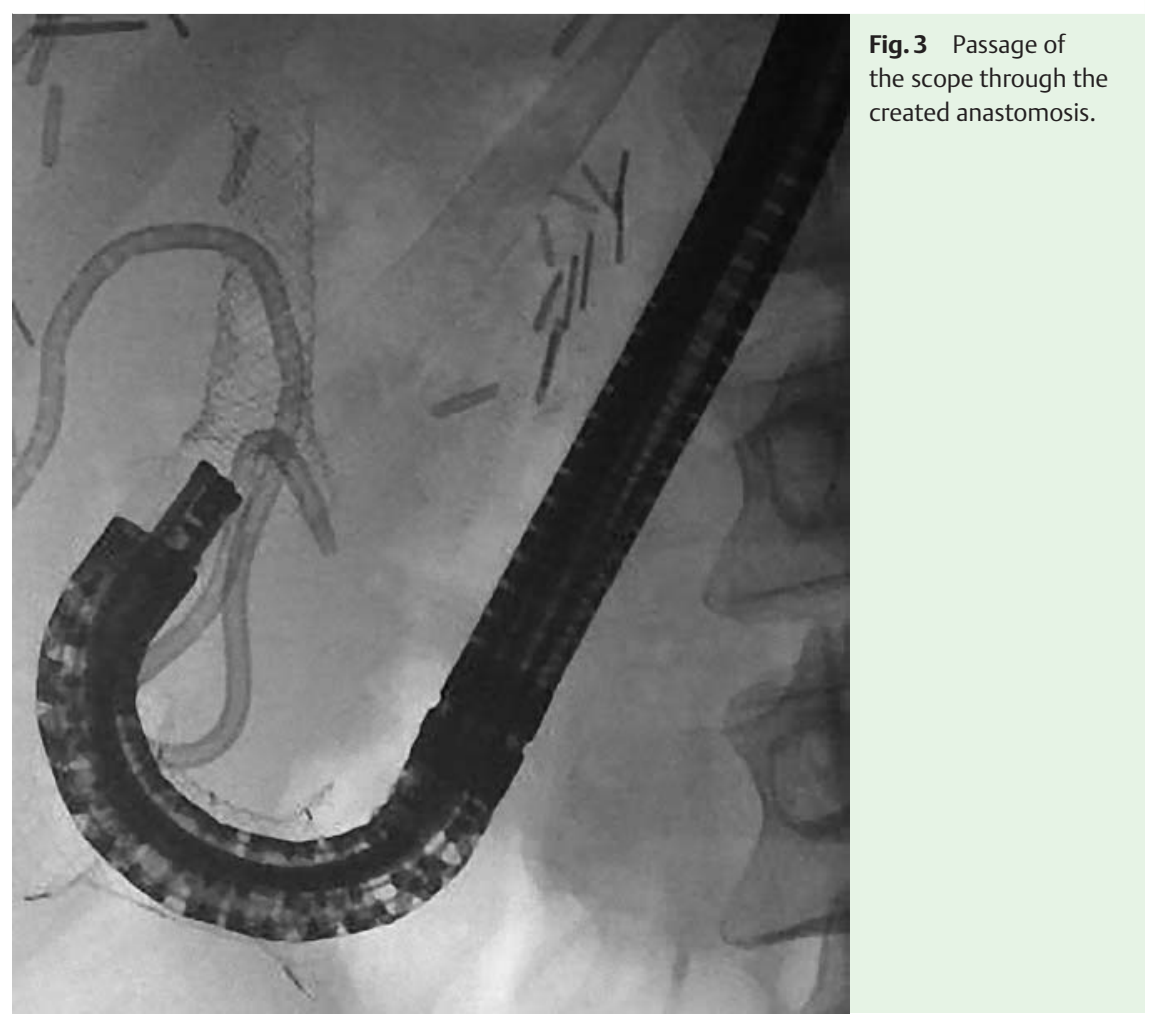

\section{Massimiliano Mutignani ${ }^{1}$,}

Raffaele Manta ${ }^{1}$, Francesco

Pugliese ${ }^{1}$, Antonio Rampoldi ${ }^{2}$, Lorenzo Dioscoridi ${ }^{1}$, Edoardo Forti ${ }^{1}$

${ }^{1}$ Diagnostic and Interventional Digestive Endoscopy, Niguarda Ca' Granda Hospital, Milan, Italy

${ }^{2}$ Interventional Radiology Unit, Niguarda Ca' Granda Hospital, Milan, Italy

\section{References}

1 Chan SM, Teoh AY. Endoscopic ultrasoundguided biliary drainage: a review. Curr Treat Options Gastroenterol 2015; 13: 171-184

2 Siripun A, Sripongpun P, Ovartlarnporn B. Endoscopic ultrasound-guided biliary intervention in patients with surgically altered anatomy. World J Gastrointest Endosc 2015; 7: 283-289

3 Ogura T, Higuchi K. Technical tips of endoscopic ultrasound-guided choledochoduodenostomy. World J Gastroenterol 2015; 21: $820-828$

4 Jürgensen C, Wentrup R, Zeitz M. Endoscopic ultrasound (EUS)-guided transduodenal drainage of an obstructed jejunal loop after hepaticojejunostomy as treatment for recurrent biliary sepsis. Endoscopy 2013; 45 (Suppl. 02): E40-E41

Bibliography

DOI http://dx.doi.org/

10.1055/s-0034-1392424

Endoscopy 2015; 47: E350-E351

(c) Georg Thieme Verlag KG

Stuttgart · New York

ISSN 0013-726X

Corresponding author

Massimiliano Mutignani, MD

Endoscopy Unit

Niguarda Ca' Granda Hospital

Piazza dell'Ospedale Maggiore 3

20162 Milan

Italy

Fax: +39-02-64442911

massimiliano.mutignani@ospedaleniguarda.it 